\title{
SOME ASYMPTOTIC THEOREMS FOR ABSTRACT DIFFERENTIAL EQUATIONS
}

\section{S. ZAIDMAN ${ }^{1}$}

Abstract. We consider in this paper results on differential equations with time independent operators; uniqueness of solutions which are bounded in the Stepanoff norm as well as weak almostperiodic solutions are some of the topics here considered.

Introduction. In this paper, which is closely related with some of our previous publications, a number of results concerning differential equations in Hilbert and Banach spaces are derived. They concern asymptotic behaviour, boundedness and almost-periodicity.

1. Our first result, a very simple one, is "essentially" the Theorem 1 in [1]. Here it is given in its natural, operator case framework.

TheOREM 1. Let $H$ be a Hilbert space, $A$ a closed linear operator in $H$ with dense domain $D(A) ; A^{*}$ be its adjoint operator, and suppose that for a real $\beta$ the relations

$$
\begin{array}{rlrl}
\operatorname{Re}(A x, x) & \leqq \beta(x, x), & & \forall x \in D(A), \\
\operatorname{Re}\left(A^{*} y, y\right) \leqq \beta(y, y), & & \forall y \in D\left(A^{*}\right)
\end{array}
$$

are verified. Let $u(t), t \geqq 0 \rightarrow D(A)$, be a strong solution of equation

$$
u^{\prime}(t)=A u(t) \text {. }
$$

Then $\|u(t)\| \leqq e^{\beta t}\|u(0)\|$ holds, $\forall t \geqq 0$.

Proof. Let us put $v(t)=e^{-\beta t} u(t)$. Then $v^{\prime}(t)=(A-\beta I) v(t)$. It is easy to see that $A-\beta I$ is the infinitesimal generator of a strongly continuous one-parameter semigroup $T_{\beta}(t)$ such that $\left\|T_{\beta}(t)\right\| \leqq 1$. We see also the representation $v(t)=T_{\beta}(t) v(0)$; consequently $\|v(t)\|$ $\leqq\|v(0)\|=\|u(0)\|$ and $\|u(t)\| \leqq e^{\beta t}\|u(0)\|$.

Now, a very simple result about asymptotic behaviour (cf. Theorem 2 in $[1])$ is the

Received by the editors September 22, 1969.

AMS Subject Classifications. Primary 3495.

Key Words and Phrases. Hilbert space, linear closed operator, adjoint operator, strong solution, infinitesimal operator, one-parameter semigroup, Banach space, almost-periodic functions, compact operators, dual Banach space, space $\mathrm{L}^{p}\left(R^{n}\right)$, truncation operator, translation operator.

${ }^{1}$ Supported by National Research Council of Canada. 
THEOREM 2. Let us have (1.1) with $\beta<0$. Then if $f_{0} \in H$ is given and $u(t), t \geqq 0 \rightarrow D(A)$ is solution of

$$
u^{\prime}(t)=A u(t)+f_{0} .
$$

There exists $w_{0} \in H$ such that $\lim _{t \rightarrow \infty} u(t)=w_{0}$.

Proof. It follows easily that $A^{-1}$ exists and belongs to $\mathcal{L}(H, H)$. We consider $w_{0}=-A^{-1} f_{0}$, and put $v(t)=u(t)-w_{0}$. We have $v^{\prime}(t)$ $=A v(t)$; apply Theorem 1 and we get, as $\beta<0, \lim _{i \rightarrow \infty} u(t)=w_{0}$.

Our next result is a simple generalization of Lemma 1 in our paper [2].

Theorem 3. Let $\mathfrak{X}$ be a Banach space, and $T_{t} \in \mathfrak{L}(\mathfrak{X}, \mathfrak{X}), t \geqq 0$, be a one parameter strongly continuous semigroup, such that $\left\|T_{t}\right\| \leqq M e^{\beta t}, \beta<0$, $t \geqq 0$. Let $A$ be its infinitesimal generator and $u(t),-\infty<t<+\infty \rightarrow D(A)$, be a strong solution of $u^{\prime}(t)=A u(t)$. Then if $\sup _{t \in R^{1}} \int_{t}^{t+1}\|u(\sigma)\|^{2} d \sigma<\infty$ it follows $u(t)=\theta$ for every real $t$.

REMARK. Similar results are given in our paper [3].

Proof. We see firstly the representation: $u(t)=T_{t-t_{0}} u\left(t_{0}\right), \forall t \geqq t_{0}$.

Then we remark existence of a sequence $\left(t_{n}\right)_{1}^{\infty}$ such that $\lim _{n \rightarrow \infty} t_{n}=-\infty$ and such that $\sup _{n} \in \mathfrak{N}\left\|u\left(t_{n}\right)\right\|=L<\infty$. Next for arbitrary real $t$ we take $n$ large enough in order to have $t_{n}<t$ and consequently $u(t)=T_{t-t_{n}} u\left(t_{n}\right)$. So we derive $\|u(t)\| \leqq M e^{\beta\left(t-t_{n}\right)} \cdot L$; for $n \rightarrow \infty$ we get $u(t)=0$.

2. In this section we give a complement to our result on almostperiodicity of certain relatively-compact valued vector functions (see [4]) by taking into account weakly almost-periodic solutions (of abstract differential equations). Remember that if $\mathfrak{X}$ is a Banach space and $\mathfrak{X}^{*}$ its strong dual, a continuous function $f(t),-\infty<t<+\infty \rightarrow \mathfrak{X}$ is weakly almost-periodic when $\left\langle x^{*}, f(t)\right\rangle$ is Bohr-almost-periodic for every $x^{*} \in \mathfrak{X}^{*}$. Our result is the following

THEOREM 4. In the Banach space $\mathfrak{X}$, consider a strongly continuous one-parameter semigroup $T_{t} \in \mathcal{L}(\mathfrak{X}, \mathfrak{X})$ such that $\lim _{t \rightarrow \infty} T_{t} x=\theta, \forall x \in \mathfrak{X}$. Let also $Q \in \mathcal{L}(\mathfrak{X}, \mathfrak{X})$ be a compact operator commuting with $T_{t}, \forall t \geqq 0$. Its inverse $Q^{-1}$ exists on a dense set in $\mathfrak{X}$, and the adjoint $\left(Q^{-1}\right)^{*}$ is defined on a dense set in $\mathfrak{X}^{*}$. Let $A$ be the infinitesimal generator of $T_{t}$; $f(t)$ a continuous weakly almost-periodic function $-\infty<t<+\infty \rightarrow \mathfrak{X}$; $u(t)$ a strong solution, on the whole real axis of equation $u^{\prime}(t)=A u(t)$ $+f(t)$, such that $\sup _{t \in R^{1}}\|u(t)\|<\infty$. Then $u(t)$ is weakly almost-periodic.

Proof. We remark first, as a standard result, the representation formula 


$$
u(t)=T_{t-t_{0}} u\left(t_{0}\right)+\int_{t_{0}}^{t} T_{t-8} f(s) d s, \quad t \geqq t_{0} .
$$

Next we see that: if $g(t),-\infty<t<+\infty \rightarrow \mathfrak{X}$, is a bounded function such that $\left\langle x^{*}, g(t)\right\rangle$ is almost-periodic for a dense set of elements in the dual space $\mathfrak{X}^{*}$, then $g(t)$ is weakly almost-periodic. The result is a corollary of the fact that uniform convergent on $R^{1}$ sequences of almost-periodic functions have almost periodic limit. A simple remark now is that $w(t)=Q u(t)$ has representation

$$
w(t)=T_{t-t_{0}} w\left(t_{0}\right)+\int_{t_{0}}^{t} T_{t-s}(Q f)(s) d s, \quad t \geqq t_{0}
$$

and that range of $w(t)$ is relatively compact in $\mathfrak{X}$ as $t \in R^{1}$. Then we have

LEMma. If $h(t)$ is continuous weakly almost-periodic, $t \in R^{1} \rightarrow \mathfrak{X}$, and if $Q$ is a compact operator $\in \mathscr{L}(\mathfrak{X}, \mathfrak{X})$ then $Q h$ is strongly almost-periodic.

In fact $h(t)$ is bounded, hence $Q h$ has relatively compact range. Moreover $Q h(t)$ is weakly almost-periodic too; by well-known facts $Q h(t)$ is strongly almost-periodic.

By this Lemma, $Q f$ is almost-periodic. We apply our Theorem 1 in [4] and obtain that $w(t)=Q u(t)$ is almost-periodic. Then $u(t)$ $=Q^{-1} w(t)=Q^{-1} Q u(t)$. We take now $x^{*} \in D\left(\left(Q^{-1}\right)^{*}\right)$ (which is dense in $\left.\mathfrak{X}^{*}\right)$.

We have $\left\langle x^{*}, Q^{-1} Q u(t)\right\rangle=\left\langle\left(Q^{-1}\right)^{*} x^{*}, w(t)\right\rangle$ which is almost-periodic Bohr. From the above made remarks, $u(t)$ is weakly almost-periodic.

3. Here we remember a certain natural generalization of almostperiodic functions.

Definition 3.1. Let $h(t), 0 \leqq t<\infty$, be a continuous function with values in the Banach space $\mathfrak{X}$. We say that $h(t)$ is in the class $\mathbb{B}_{\mathfrak{X}}^{+}$when the set of translates $(h(t+\eta))_{\eta \geq 0}$ is a relatively compact set in the space $C[0, \infty ; \mathfrak{X}]$.

Definition 3.2. Let $h(t)$ be a continuous function, $0 \leqq t<\infty \rightarrow \mathfrak{X}$. We say that $h(t)$ is in the class $\mathfrak{F}_{\mathfrak{X}}^{+}$when $\forall \epsilon>0, \exists L_{\epsilon}>0, N_{\epsilon}>0$, such that in every interval $[a, a+L] \subset[0, \infty), \exists \zeta_{\epsilon}$ with property

$$
\sup _{t>N_{\epsilon}}\left\|h\left(t+\zeta_{\epsilon}\right)-h(t)\right\|_{\mathfrak{X}}<\epsilon
$$

In the Appendix of our paper [1] a proof of the inclusion $B_{\mathfrak{X}}^{+} \subset \mathcal{F}_{\mathfrak{X}}^{+}$is indicated.

Definition 3.3. A continuous function, $0 \leqq t<\infty \rightarrow \mathfrak{X}, h(t)$ is called weakly- $B_{\mathfrak{X}}^{+}\left(\right.$resp. weakly $-\mathcal{F}_{\mathfrak{X}}^{+}$) if, for each $x^{*} \in \mathfrak{X}^{*},\left\langle x^{*}, h(t)\right\rangle$ is in 
class $\mathbb{B}^{+}\left(\right.$resp $\left.\mathfrak{F}^{+}\right)$corresponding to $\mathfrak{X}=$ scalar field. It is easy to see that if $g(t) \in \mathcal{F}_{\mathfrak{X}}^{+}$, then, $\forall x^{*} \in \mathfrak{X}^{*},\left\langle x^{*}, g(t)\right\rangle \in \mathcal{F}^{+}$. Also, we have the standard proof of the fact that uniform limits on $0 \leqq t<\infty$ of sequences $\left(h_{n}(t)\right)_{1}^{\infty} \subset \mathcal{F}_{\mathfrak{x}}^{+}$belong to the same class. We do now a simple observation, connected with Theorem 5 in [1]. We have precisely the

Theorem 5. Let $\mathfrak{X}$ be a Banach space; $T_{t}, t \geqq 0 \rightarrow \mathfrak{L}(\mathfrak{X}, \mathfrak{X})$, be a strongly continuous one-parameter semigroup, such that $\left\|T_{t}\right\| \leqq M, t \geqq 0$. Let $A$ be its infinitesimal generator and $u(t), t \geqq 0 \rightarrow D(A)$, be a strong solution of the equation: $u^{\prime}(t)=A u(t), t \geqq 0$. Suppose that $u(t)$ has relatively compact trajectory; then $u(t) \in \mathcal{F}_{\mathfrak{z}}^{+}$.

Proof. We have as usual, representation $u(t)=T_{t} u(0), t \geqq 0$. We prove that $u(t) \in \beta_{x}^{+}$. Consider the set of vector-functions: $\{u(t+\eta)\}_{\eta \geqq 0}=\left\{T_{t+\eta} u(0)\right\}_{\eta \geqq 0}$. By relative compactness we may find a sequence $\left(\eta_{n}\right)_{1}^{\infty} \subset[0, \infty)$ such that $\left(T_{\eta_{n}} u(0)\right)_{n=1}^{\infty}$ is a Cauchy sequence in $\mathfrak{X}$. Then $\left\{u\left(t+\eta_{n}\right)\right\}_{n=1}^{\infty}$ is a Cauchy sequence in $C[0, \infty ; \mathfrak{X}]$. This follows from the obvious estimate:

$$
\begin{aligned}
\left\|T_{t+\eta_{n}} u(0)-T_{t+\eta_{m}} u(0)\right\| & \leqq\left\|T_{t}\right\|\left\|T_{\eta_{n}} u(0)-T_{\eta_{m}} u(0)\right\| \\
& \leqq M\left\|u\left(\eta_{n}\right)-u\left(\eta_{m}\right)\right\| .
\end{aligned}
$$

We complement this result by another one, on weak- $\mathcal{F}_{\mathfrak{x}}^{+}$solutions.

TheOREM 6. Let $\mathfrak{X}$ be a Banach space; $T_{t}, t \geqq 0 \rightarrow \mathfrak{L}(\mathfrak{X}, \mathfrak{X})$, be a strongly continuous one-parameter semigroup such that $\left\|T_{t}\right\| \leqq M, t \geqq 0$. Let $A$ be its infinitesimal generator; suppose that for a complex $\lambda_{0}$, operator $\left(\lambda_{0}-A\right)^{-1}$ is a linear compact operator in $\mathfrak{X}$; suppose also the adjoint operator $A^{*}$ be densely defined in $\mathfrak{X}^{*}$. Consider then $u(t), t \geqq 0 \rightarrow D(A)$ a strong solution of $u^{\prime}(t)=A u(t), t \geqq 0$, such that $\|u(t)\| \leqq M, t \geqq 0$. Then $u(t)$ is weakly-F $\mathcal{F}_{\mathfrak{X}}^{+}$.

Proof. We have again: $u(t)=T_{t} u(0), t \geqq 0$. Denote by $v(t)$ the vector-function $\left(\lambda_{0}-A\right)^{-1} u(t)$. Because $T_{t}$ commutes with $\left(\lambda_{0}-A\right)^{-1}$ we obtain $v(t)=T_{t} v(0)$, and moreover $v(t)$ has relatively compact trajectory. We apply the previous theorem and get $v(t) \in \mathcal{F}_{\mathfrak{x}}^{+}$. Hence $u(t)=\left(\lambda_{0}-A\right) v(t)$. Take then $x^{*} \in D\left(A^{*}\right)$. We have $\left\langle x^{*}, u(t)\right\rangle$ $=\left\langle x^{*}, \quad\left(\lambda_{0}-A\right) v(t)\right\rangle=\left\langle\left(\lambda_{0}-A\right)^{*} x^{*}, v(t)\right\rangle=\left\langle y^{*}, v(t)\right\rangle$. Applying the previous remarks our result follows.

We end this paper giving, in a concrete case an effective criterium in order that for a given semigroup $T_{t}$, the trajectory $\left\{T_{t} x\right\}_{t \geq 0}$ be relatively compact (see Theorem 5 ). Consider the space $L^{p}\left(R^{p}\right)$, $1 \leqq p<\infty$. Remember a necessary and sufficient condition for a set $a \subset L^{p}\left(R^{n}\right)$ to be relatively compact:

(i) $\int_{R^{n}}|u(x)|{ }^{p} d x \leqq M, \forall u \in Q$, 
(ii) $\lim _{\rho \rightarrow \infty} \int_{R^{n}}\left|u_{\rho}(x)\right|^{p} d x=0$ uniformly on $u \in Q$; here $u_{\rho}(x)=u(x)$, $|x|>\rho$ and $u_{\rho}(x)=0$ for $|x| \leqq \rho$.

Call $t_{\rho} ; \phi \rightarrow \phi_{\rho}, L^{p} \rightarrow L^{p}$, the truncation operator,

(iii) $\lim _{|h| \rightarrow 0} \int_{R^{n}}\left|\left(\zeta_{h} u-u\right)(x)\right|^{p} d x=0$, uniformly on $u \in Q$. Here $\left(\zeta_{h} u\right)(x)=u(x+h)$ is the translation operator. Then we have

Theorem 7. Let $T_{t}, t \geqq 0 \rightarrow \mathfrak{L}\left(L^{p}\left(R^{n}\right), L^{p}\left(R^{n}\right)\right), 1 \leqq p<\infty$, be a strongly continuous semigroup such that $\left\|T_{t}\right\| \leqq M, t \geqq 0$. Suppose that $T_{t}$ commutes with the truncation operator $t_{p}$, for each $\rho>0$ and with the translation operator $\zeta_{h}$ for each $h \in R^{n}$. Then the set $a=\left\{T_{t} \phi_{0}\right\}_{t 30}$ is, for fixed $\phi_{0} \in L^{p}\left(R^{n}\right)$, a relatively compact set in $L^{p}\left(R^{n}\right)$.

The proof is immediate if we apply the previous criterium.

\section{REFERENCES}

1. S. Zaidman, Théorèmes qualitatifs pour des équations aux dérivêes partielles, Acad. R. P. Romîne Stud. Cerc. Mat. 6 (1955), 645-666. (Roumanian) MR 17, 1211.

2. - Uniqueness of bounded solutions for some abstract differential equations, Ann. Univ. Ferrara Sez. VII (to appear).

3. - Bounded solutions of some abstract differential equations, Proc. Amer. Math. Soc. 23 (1969), 340-342.

4. - On some almost-periodic functions, Ann. Univ. Ferrara Sez. VII (to appear).

University of Montreal, Montreal, Quebec, Canada 